\title{
An $(l, \kappa)$-fuzzy $M$ closed and $(l, \kappa)$ - generalized fuzzy $M$ closed sets in double fuzzy topological spaces
}

\author{
J. Sathiyaraj ${ }^{1 *}$, A. Vadivel ${ }^{2}$ and O. Uma Maheshwari ${ }^{3}$
}

\begin{abstract}
In double fuzzy topological spaces, $(l, \kappa)$ - fuzzy $\theta$-closed, $(l, \kappa)$-fuzzy $M$-closed sets and $(l, \kappa)$-generalized fuzzy $M$-closed sets are introduced. Also we study some of their properties.

\section{Keywords}

$(l, \kappa)$ - fuzzy $\theta$-closed, $(l, \kappa)$-fuzzy $M$-closed sets, $(l, \kappa)$-generalized fuzzy $M$-closed sets, $(l, \kappa)$-generalized fuzzy $M$-interior, $(l, \kappa)$-generalized fuzzy $M$-closure.

\section{AMS Subject Classification} 54A40, 45D05, 03E72.

1,2 Post Graduate and Research Department of Mathematics, Government Arts College (Autonomous), Karur - 639005, Tamil Nadu, India. ${ }^{3}$ Post Graduate and Research Department of Mathematics, J. J. College of Arts and Science (Autonomous), Pudukkottai - 622422, Tamil Nadu, India.

*Corresponding author: ${ }^{\text {* }}$ sjsathiyaa@gmail.com; ${ }^{2}$ avmaths@gmail.com; ${ }^{3}$ ard_uma@yahoo.com.sg.

Article History: Received 22 November 2018; Accepted 09 May 2019

(C)2019 MJM
\end{abstract}

\section{Contents}

1 Introduction and Preliminaries .284

2 An $(l, \kappa)$ - fuzzy $M$ closed sets 285

An $(l, \kappa)$-generalized fuzzy $M$ closed sets .287

References 288

\section{Introduction and Preliminaries}

"Intuitionistic fuzzy sets and Intuitionistic fuzzy topological space" were initiated by Atanassov [1] in 1993 and Coker [2] in 1997 respectively. In 2005, Garcia and Rodabaugh [7] coined the term "double" instead of "intuitionistic". In the past two decades many researchers [9-11, 16] doing more applications on double fuzzy topological spaces. From 2011, El-Maghrabi and Al - Johany [3-6] introduced and studied some properties on $M$-open sets and maps in topological spaces. Recently these kind of sets were studied in Ŝstak's Fuzzy topological spaces [14]. In this paper we introduce $(\imath, \kappa)$ - fuzzy $\theta$-closed, $(\imath, \kappa)$-fuzzy $M$-closed sets, $(\imath, \kappa)$ generalized fuzzy $M$ - closed sets and study some of their properties in double fuzzy topological spaces.

$X$ denotes a non-empty set, $I=[0,1], I_{0}=(0,1], I_{1}=$ $[0,1), \quad \underline{0}(X)=0, \underline{1}(X)=1, \quad \imath \in I_{0} \& \kappa \in I_{1}$ and always $\imath+\kappa \leq 1$. $I^{X}$ is a family of all fuzzy sets on $X$. In 2002 Samanta and Mondal[13] defined the double fuzzy topological space (briefly, dfts) denoted by $\left(X, \eta, \eta^{*}\right)$, also defined $(\imath, \kappa)$ - fuzzy open (resp. $(\boldsymbol{l}, \kappa)$-fuzzy closed) (briefly $(\boldsymbol{l}, \kappa)$ - $f o$ (resp. $(\boldsymbol{\imath}, \kappa)-f c)$ ) set if $\eta(\gamma) \geq \imath$ and $\eta^{*}(\gamma) \leq \kappa$, (resp. $1-\gamma$ is an $(\boldsymbol{\imath}, \kappa)$ - $f o$ set.) The double fuzzy interior and double fuzzy closure operators [8] are defined from $I^{X} \times I_{0} \times I_{1} \rightarrow I^{X}$ as follows

$$
\begin{aligned}
I_{\eta, \eta^{*}}(\gamma, \imath, \kappa) & =\bigvee\left\{v \in I^{X} \mid v \leq \gamma, \eta(v) \geq \imath, \eta^{*}(v) \leq \kappa\right\} \\
C_{\eta, \eta^{*}}(\gamma, \imath, \kappa) & = \\
\bigwedge\{v & \left.\in I^{X} \mid v \geq \gamma, \eta(\underline{1}-v) \geq \imath, \eta^{*}(\underline{1}-v) \leq \kappa\right\}
\end{aligned}
$$

$\gamma \in I^{X}$, is called $(\boldsymbol{\imath}, \kappa)$-fuzzy regular open [12] (resp. $(l, \kappa)$ fuzzy regular closed)(briefly $(\boldsymbol{\imath}, \kappa)$-fro (resp. $(\boldsymbol{\imath}, \kappa)-f r c))$ set if $\gamma=I_{\eta, \eta^{*}}\left(C_{\eta, \eta^{*}}(\gamma, \imath, \kappa), \boldsymbol{\imath}, \kappa\right)$ (resp. $\underline{1}-\gamma$ is $(\boldsymbol{\imath}, \kappa)$-fro set.) The operators $\delta C_{\eta, \eta^{*}}$ and $\delta I_{\eta, \eta^{*}:} I^{X} \times I_{0} \times I_{1} \rightarrow I^{X}[11]$ as follows

$$
\begin{aligned}
& \delta I_{\eta, \eta^{*}}(\gamma, \imath, \kappa)=\bigvee\left\{v \in I^{X} \mid v \leq \gamma, v \text { is an }(\imath, \kappa)-f r o\right\} \\
& \delta C_{\eta, \eta^{*}}(\gamma, \imath, \kappa)=\bigwedge\left\{v \in I^{X} \mid v \geq \gamma, v \text { is an }(\imath, \kappa)-f r c\right\}
\end{aligned}
$$

A fuzzy set $\gamma \in I^{X}$, is called $(\boldsymbol{\imath}, \kappa)$-fuzzy $\delta$ pre open (resp. $(\boldsymbol{l}, \kappa)$ - fuzzy $e$ open, $(\boldsymbol{\imath}, \kappa)$-fuzzy $\delta$ pre closed and $(\boldsymbol{\imath}, \kappa)$ fuzzy $e$ closed ) (briefly $(\imath, \kappa)-f \delta p o$ (resp. $(\imath, \kappa)$-feo, $(\imath, \kappa)$ $f \delta p c$ and $(\imath, \kappa)-f e c))$ [11]set if $\gamma \leq I_{\eta, \eta^{*}}\left(\delta C_{\eta, \eta^{*}}(\gamma, \imath, \kappa)\right.$, $\imath, \kappa)\left(\operatorname{resp} . \quad \gamma \leq C_{\eta, \eta^{*}}\left(\delta I_{\eta, \eta^{*}}(\gamma, \imath, \kappa), \imath, \kappa\right) \vee I_{\eta, \eta^{*}}\left(\delta C_{\eta, \eta^{*}}\right.\right.$ $(\gamma, \imath, \kappa), \imath, \kappa), \underline{1}-\gamma$ is an $(\imath, \kappa)-f \delta p o$ and $\underline{1}-\gamma$ is an $(\imath, \kappa)-$ $f e o)$. The operators $\delta P C_{\eta, \eta^{*}}, \delta P I_{\eta, \eta^{*}}, e C_{\eta, \eta^{*}}$ and $e I_{\eta, \eta^{*}}$ : 


$$
\begin{aligned}
I^{X} \times I_{0} \times I_{1} \rightarrow I^{X}[11] \text { defined as } & \\
\delta P I_{\eta, \eta^{*}}(\gamma, \imath, \kappa) & =\bigvee\left\{v \in I^{X} \mid v \leq \gamma, v \text { is an }(l, \kappa)-f \delta p o\right\}, \\
\delta P C_{\eta, \eta^{*}}(\gamma, \imath, \kappa) & =\bigwedge\left\{v \in I^{X} \mid v \geq \gamma, v \text { is an }(l, \kappa)-f \delta p c\right\}, \\
e I_{\eta, \eta^{*}}(\gamma, \imath, \kappa) & =\bigvee\left\{v \in I^{X} \mid v \leq \gamma, v \text { is an }(l, \kappa)-f e o\right\}, \\
e C_{\eta, \eta^{*}}(\gamma, \imath, \kappa) & =\bigwedge\left\{v \in I^{X} \mid v \geq \gamma, v \text { is an }(l, \kappa)-f e c\right\}
\end{aligned}
$$

All other notations are from fuzzy set theory, which is given by Zadeh [15] and his followers.

\section{An $(l, \kappa)$ - fuzzy $M$ closed sets}

Definition 2.1. Let $\left(X, \eta, \eta^{*}\right)$ be a dfts, $\forall \gamma, v \in I^{X}$, the operators $(l, \kappa)$ - fuzzy $\theta$ interior and $(l, \kappa)$ - fuzzy $\theta$ closure denoted by $(l, \kappa)-\theta I_{\eta, \eta^{*}}$ and $(l, \kappa)-\theta C_{\eta, \eta^{*}}: I^{X} \times I_{0} \times I_{1} \rightarrow I^{X}$ are defined as

$$
\begin{aligned}
& \theta I_{\eta, \eta^{*}}(\gamma, \imath, \kappa)= \\
& \quad \bigvee\left\{I_{\eta, \eta^{*}}(v, l, \kappa) \mid v \leq \gamma, \eta(\underline{1}-v) \geq \imath \& \eta^{*}(\underline{1}-v) \leq \kappa\right\}
\end{aligned}
$$$$
\text { and }
$$$$
\theta C_{\eta, \eta^{*}}(\gamma, \iota, \kappa)=
$$$$
\bigwedge\left\{C_{\eta, \eta^{*}}(v, \imath, \kappa) \mid v \geq \gamma, \eta(v) \geq \imath \& \eta^{*}(v) \leq \kappa\right\} .
$$

Definition 2.2. In a dfts $\left(X, \eta, \eta^{*}\right), \gamma \in I^{X}$ is called an

1. $(l, \kappa)$ - fuzzy $\theta$ open (resp. $(l, \kappa)$ - fuzzy $\theta$ semi open) (briefly $(l, \kappa)-f \theta o$ (resp. $(l, \kappa)-f \theta s o))$ set if $\gamma=\theta I_{\eta, \eta^{*}}(\gamma, \imath, \kappa)$. (resp.

$\left.\gamma \leq C_{\eta, \eta^{*}}\left(\theta I_{\eta, \eta^{*}}(\gamma, l, \kappa), l, \kappa\right).\right)$

2. $(l, \kappa)$ - fuzzy $\theta$ closed (resp. $(l, \kappa)$ - fuzzy $\theta$ semi closed) (briefly $(\boldsymbol{l}, \kappa)-f \theta c$ (resp. $(l, \kappa)-f \theta c)$ ) set if $\underline{1}-\gamma$ is an $(l, \kappa)-f \theta o$ (resp. $(l, \kappa)-f \theta s o)$ set.

Definition 2.3. In a dfts $\left(X, \eta, \eta^{*}\right), \gamma \in I^{X}$ is called an

1. $(\boldsymbol{l}, \kappa)$ - fuzzy $M$ closed (briefly $(\boldsymbol{l}, \kappa)-f M c)$ set if $\gamma \geq$ $I_{\eta, \eta^{*}}\left(\theta C_{\eta, \eta^{*}}(\gamma, l, \kappa), \imath, \kappa\right) \wedge C_{\eta, \eta^{*}}\left(\delta I_{\eta, \eta^{*}}(\gamma, l, \kappa), \imath, \kappa\right)$.

2. $(l, \kappa)$ - fuzzy $M$ open (briefly $(l, \kappa)-f M o$ ) set if $\underline{1}-\gamma$ is an $(\imath, \kappa)-f M c$ set.

Definition 2.4. Let $\left(X, \eta, \eta^{*}\right)$ be a dfts, then the

1. union of all $(l, \kappa)$-fMo (resp. $(l, \kappa)-f \theta o$ and $(l, \kappa)$ $f \theta s o)$ sets contained in $\gamma$ is called the $(\imath, \kappa)$-fuzzy $M$ (resp. $(l, \kappa)$-fuzzy $\theta$ and $(l, \kappa)$-fuzzy $\theta$ semi) interior of $\gamma$ and is denoted by $M I_{\eta, \eta^{*}}(\gamma, l, \kappa)$ (resp. $\theta I_{\eta, \eta^{*}}$ $(\gamma, \imath, \kappa)$ and $\left.\theta s I_{\eta, \eta^{*}}(\gamma, \imath, \kappa)\right)$.

2. intersection of all $(l, \kappa)-f M c$ (resp. $(l, \kappa)-f \theta c$ and $(l, \kappa)-f \theta s c)$ sets containing $\gamma$ is called the $(\imath, \kappa)$-fuzzy $M$ (resp. $(l, \kappa)$-fuzzy $\theta$ and $(l, \kappa)$-fuzzy $\theta$ semi) closure of $\gamma$ and is denoted by $M C_{\eta, \eta^{*}}(\gamma, \imath, \kappa)$ (resp. $\theta C_{\eta, \eta^{*}}(\gamma, \imath, \kappa)$ and $\left.\theta s C_{\eta, \eta^{*}}(\gamma, \imath, \kappa)\right)$.
Proposition 2.5. In a dfts $\left(X, \eta, \eta^{*}\right), \forall \gamma, v \in I^{X}$,

(a) $M I_{\eta, \eta^{*}}(\underline{0}, \imath, \kappa)=\underline{0}$, and $M I_{\eta, \eta^{*}}(\underline{1}, \imath, \kappa)=\underline{1}$.

(b) $M C_{\eta, \eta^{*}}(\underline{0}, \imath, \kappa)=\underline{0}$, and $M C_{\eta, \eta^{*}}(\underline{1}, l, \kappa)=\underline{1}$.

(c) $\underline{1}-M I_{\eta, \eta^{*}}(\gamma, \imath, \kappa)=M C_{\eta, \eta^{*}}(\underline{1}-\gamma, l, \kappa)$.

(d) $\underline{1}-M C_{\eta, \eta^{*}}(\gamma, \imath, \kappa)=M I_{\eta, \eta^{*}}(\underline{1}-\gamma, \imath, \kappa)$.

(e) If $\gamma<v$ then $M I_{\eta, \eta^{*}}(\gamma, \imath, \kappa)<M I_{\eta, \eta^{*}}(v, \imath, \kappa)$.

(f) If $\gamma \leq v$ then $M C_{\eta, \eta^{*}}(\gamma, \imath, \kappa) \leq M C_{\eta, \eta^{*}}(v, \imath, \kappa)$.

(g) $M I_{\eta, \eta^{*}}(\gamma, \imath, \kappa) . \leq \gamma \leq M C_{\eta, \eta^{*}}(\gamma, \imath, \kappa)$.

(h) $M C_{\eta, \eta^{*}}(\gamma \vee \nu, \imath, \kappa) \geq M C_{\eta, \eta^{*}}(\gamma, \imath, \kappa) \vee M C_{\eta, \eta^{*}}(\nu, \imath, \kappa)$.

(i) $M C_{\eta, \eta^{*}}(\gamma \wedge v, \imath, \kappa) \leq M C_{\eta, \eta^{*}}(\gamma, \imath, \kappa) \wedge M C_{\eta, \eta^{*}}(\nu, \imath, \kappa)$.

(j) $M C_{\eta, \eta^{*}}\left(M C_{\eta, \eta^{*}}(\gamma, \imath, \kappa), \iota, \kappa\right)=M C_{\eta, \eta^{*}}(\gamma, \imath, \kappa)$.

(k) If $\gamma$ is $(\imath, \kappa)-f M c$ set then $M C_{\eta, \eta^{*}}(\gamma, \imath, \kappa)=\gamma$.

(l) If $v$ is $(\imath, \kappa)-f M o$ set then $v q \gamma$ iff $v q M C_{\eta, \eta^{*}}(\gamma, \imath, \kappa)$.

(m) $M I_{\eta, \eta^{*}}(\gamma \vee v, \imath, \kappa) \geq M I_{\eta, \eta^{*}}(\gamma, \imath, \kappa) \vee M I_{\eta, \eta^{*}}(v, \imath, \kappa)$.

(n) $M I_{\eta, \eta^{*}}(\gamma \wedge v, \imath, \kappa) \leq M I_{\eta, \eta^{*}}(\gamma, \imath, \kappa) \wedge M I_{\eta, \eta^{*}}(\nu, \imath, \kappa)$.

(o) $M I_{\eta, \eta^{*}}\left(M I_{\eta, \eta^{*}}(\gamma, \imath, \kappa), \imath, \kappa\right)=M I_{\eta, \eta^{*}}(\gamma, \imath, \kappa)$.

(p) If $\gamma$ is $(\imath, \kappa)-f M o$ set then $M I_{\eta, \eta^{*}}(\gamma, \imath, \kappa)=\gamma$.

(q) $\gamma \leq C_{\eta, \eta^{*}}(\gamma, \imath, \kappa) \leq \delta C_{\eta, \eta^{*}}(\gamma, \imath, \kappa) \leq \theta C_{\eta, \eta^{*}}(\gamma, \imath, \kappa)$.

(r) $\theta I_{\eta, \eta^{*}}(\gamma, \imath, \kappa) \leq \delta I_{\eta, \eta^{*}}(\gamma, \imath, \kappa) \leq I_{\eta, \eta^{*}}(\gamma, l, \kappa) \leq \gamma$.

Theorem 2.6. In any dfts $\left(X, \eta, \eta^{*}\right)$, Every

1. $(\boldsymbol{l}, \kappa)-f \theta s c$ (resp. $(\boldsymbol{l}, \kappa)-f \delta p c)$ set is an $(\boldsymbol{l}, \kappa)-f M c$ set.

2. $(l, \kappa)-f \theta c$ set is an $(l, \kappa)-f \theta s c$ set.

3. $(\boldsymbol{l}, \kappa)-f \theta c$ set is an $(\boldsymbol{\imath}, \kappa)-f c$ set.

4. $(\boldsymbol{l}, \kappa)-f c$ set is an $(l, \kappa)-f \delta p c$ set.

5. $(\boldsymbol{\imath}, \boldsymbol{\kappa})-f M c$ set is an $(\boldsymbol{\imath}, \boldsymbol{\kappa})-f e c$ set.

Remark 2.7. The converse of the above theorem, in general, need not be true. It can be verified from the following examples.

Example 2.8. Consider the $\operatorname{dfts}\left(X, \eta, \eta^{*}\right)$ with $X=\{a, b, c\}$ and

$\eta(\gamma)=\left\{\begin{array}{ll}1, & \text { if } \gamma \in\{\underline{0}, \underline{1}\}, \\ \frac{1}{2}, & \text { if } \gamma=\underline{0.1} \\ 0, & \text { Otherwise. }\end{array} \quad \eta^{*}(\gamma)= \begin{cases}0, & \text { if } \gamma \in\{\underline{0}, \underline{1}\} \\ \frac{1}{2}, & \text { if } \gamma=\underline{0.1} \\ 1, & \text { Otherwise. }\end{cases}\right.$

Then the fuzzy set $\underline{0.1}$ is an $\left(\frac{1}{2}, \frac{1}{2}\right)-f M c$ set but not an $\left(\frac{1}{2}, \frac{1}{2}\right)$ $f \delta p c$ set. 
Example 2.9. Consider the $\operatorname{dfts}\left(X, \eta, \eta^{*}\right)$ with $X=\{a, b, c\}$ and

$\eta(\gamma)=\left\{\begin{array}{ll}1, & \text { if } \gamma \in\{\underline{0}, \underline{1}\}, \\ \frac{2}{3}, & \text { if } \gamma=\gamma_{1}, \\ \frac{1}{3}, & \text { if } \gamma=\gamma_{2}, \\ 0, & \text { Otherwise. }\end{array} \quad \eta^{*}(\gamma)= \begin{cases}0, & \text { if } \gamma \in\{\underline{0}, \underline{1}\} \\ \frac{1}{3}, & \text { if } \gamma=\gamma_{1}, \\ \frac{2}{3}, & \text { if } \gamma=\gamma_{2}, \\ 1, & \text { Otherwise }\end{cases}\right.$

where $\quad \gamma_{1}(a)=0.3, \quad \gamma_{1}(b)=0.4, \quad \gamma_{1}(c)=0.5, \quad \gamma_{2}(a)=$ $0.6, \quad \gamma_{2}(b)=0.9, \quad \gamma_{2}(c)=0.5, \gamma_{3}(a)=0.3, \gamma_{3}(b)=0$ and $\gamma_{3}(c)=0.5$. Then the fuzzy set $\gamma_{3}$ is an $\left(\frac{1}{3}, \frac{2}{3}\right)-f M c$ set but not an $\left(\frac{1}{3}, \frac{2}{3}\right)-f \theta s c$ set.

Example 2.10. Consider the $\operatorname{dfts}\left(X, \eta, \eta^{*}\right)$ with $X=\{a, b, c\}$ and

$\eta(\gamma)=\left\{\begin{array}{ll}1, & \text { if } \gamma \in\{\underline{0}, \underline{1}\}, \\ \frac{3}{4}, & \text { if } \gamma=\gamma_{1}, \\ \frac{1}{4}, & \text { if } \gamma=\gamma_{2}, \\ 0, & \text { Otherwise. }\end{array} \quad \eta^{*}(\gamma)= \begin{cases}0, & \text { if } \gamma \in\{\underline{0}, \underline{1}\} \\ \frac{1}{4}, & \text { if } \gamma=\gamma_{1}, \\ \frac{3}{4}, & \text { if } \gamma=\gamma_{2}, \\ 1, & \text { Otherwise }\end{cases}\right.$

where $\gamma_{1}(a)=0.3, \gamma_{1}(b)=0.4, \gamma_{1}(c)=0.5, \gamma_{2}(a)=0.6$, $\gamma_{2}(b)=0.5$, and $\gamma_{2}(c)=0.5$. Then the fuzzy set $\gamma_{1}$ is an $\left(\frac{1}{4}, \frac{3}{4}\right)-f \theta s c$ set but not an $\left(\frac{1}{4}, \frac{3}{4}\right)-f \theta c$ set.

Example 2.11. Consider the dfts $\left(X, \eta, \eta^{*}\right)$ with $X=\{a, b, c\}$ and

$\eta(\gamma)=\left\{\begin{array}{ll}1, & \text { if } \gamma \in\{\underline{0}, \underline{1}\}, \\ \frac{2}{5}, & \text { if } \gamma=\underline{0.5}, \\ \frac{1}{5}, & \text { if } \gamma=\gamma_{1}, \\ 0, & \text { Otherwise. }\end{array} \quad \eta^{*}(\gamma)= \begin{cases}0, & \text { if } \gamma \in\{\underline{0}, \underline{1}\} \\ \frac{3}{5}, & \text { if } \gamma=\underline{0.5} \\ \frac{4}{5}, & \text { if } \gamma=\gamma_{1}, \\ 1, & \text { Otherwise }\end{cases}\right.$

where $\gamma_{1}(a)=0.3, \gamma_{1}(b)=0.5$, and $\gamma_{1}(c)=0.5$, then the fuzzy set $\underline{1}-\gamma_{1}$ is an $\left(\frac{1}{5}, \frac{4}{5}\right)-f c$ set but not an $\left(\frac{1}{5}, \frac{4}{5}\right)-f \theta c$ set.

Example 2.12. Consider the $\operatorname{dfts}\left(X, \eta, \eta^{*}\right)$ with $X=\{a, b, c\}$ and

$\eta(\gamma)=\left\{\begin{array}{ll}1, & \text { if } \gamma \in\{\underline{0}, \underline{1}\}, \\ \frac{1}{6}, & \text { if } \gamma=\gamma_{1}, \\ 0, & \text { Otherwise. }\end{array} \quad \eta^{*}(\gamma)= \begin{cases}0, & \text { if } \gamma \in\{\underline{0}, \underline{1}\} \\ \frac{5}{6}, & \text { if } \gamma=\gamma_{1}, \\ 1, & \text { Otherwise }\end{cases}\right.$

where $\gamma_{1}(a)=0.5, \gamma_{1}(b)=0.3, \gamma_{1}(c)=0.2, \gamma_{2}(a)=0.5$, $\gamma_{2}(b)=0.6$ and $\gamma_{2}(c)=0.6$, then the fuzzy set $\gamma_{2}$ is an $\left(\frac{1}{6}, \frac{5}{6}\right)$ $f e c$ set but not an $\left(\frac{1}{6}, \frac{5}{6}\right)-f M c$ set.

From the above theorem, examples and by [11], the following implications are hold. $(l, \kappa)-f \theta c$

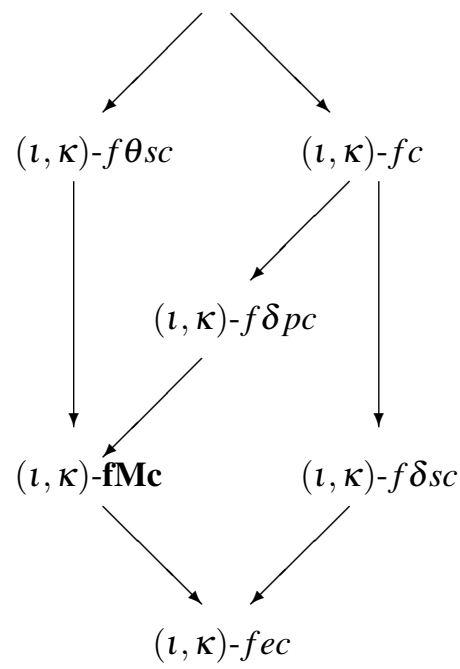

Note: $A \rightarrow B$ denotes $A$ implies $B$, but not conversely.

Theorem 2.13. Let $\left(X, \eta, \eta^{*}\right)$ be a dfts,

(i) $\bigvee_{i \in \mathrm{N}} \gamma_{i}$ is an $(l, \kappa)-f M o$ set if $\forall i \in \mathrm{N}, \gamma_{i}$ be an $(l, \kappa)$ fMo set.

(ii) $\bigwedge_{i \in \mathrm{N}} \gamma_{i}$ is an $(l, \kappa)-f M c$ set if $\forall i \in \mathrm{N}, \gamma_{i}$ be an $(l, \kappa)-f M c$ set.

Proof: (i) Let $\gamma_{i}$ be an $(l, \kappa)-f M o$ set, $\forall i \in \mathrm{N}$ then

$$
\begin{aligned}
& \gamma_{i} \subseteq C_{\eta, \eta^{*}}\left(\theta I_{\eta, \eta^{*}}\left(\gamma_{i}, l, \kappa\right), \imath, \kappa\right) \\
& \vee I_{\eta, \eta^{*}}\left(\delta C_{\eta, \eta^{*}}\left(\gamma_{i}, l, \kappa\right), \imath, \kappa\right) \quad \forall i \in \mathrm{N} \text {, } \\
& \Rightarrow \bigvee_{i \in \mathrm{N}} \gamma_{i} \subseteq \bigvee_{i \in I}\left(C_{\eta, \eta^{*}}\left(\theta I_{\eta, \eta^{*}}\left(\gamma_{i}, l, \kappa\right), \imath, \kappa\right)\right. \\
& \left.\vee I_{\eta, \eta^{*}}\left(\delta C_{\eta, \eta^{*}}\left(\gamma_{i}, \imath, \kappa\right), \imath, \kappa\right)\right) \\
& \subseteq C_{\eta, \eta^{*}}\left(\theta I_{\eta, \eta^{*}}\left(\bigvee_{i \in I} \gamma_{i}, \imath, \kappa\right), \imath, \kappa\right) \\
& \bigvee I_{\eta, \eta^{*}}\left(\delta C_{\eta, \eta^{*}}\left(\bigvee_{i \in I} \gamma_{i}, \imath, \kappa\right), \imath, \kappa\right)
\end{aligned}
$$

Thus $\bigvee_{i \in I} \gamma_{i}$ is an $(\boldsymbol{l}, \boldsymbol{\kappa})$-fMo set.

(ii) Similar to the proof of (i).

Theorem 2.14. In $\operatorname{dfts}\left(X, \eta, \eta^{*}\right)$, let $\gamma, v \in I^{X}$

(i) $\gamma \wedge v$ is an $(l, \kappa)-f M o$ set if $\gamma$ is an $(l, \kappa)$-fMo set and $\eta(v) \geq \imath, \eta^{*}(v) \leq \kappa$

(ii) $\gamma \vee v$ is an $(l, \kappa)-f M c$ set if $\gamma$ is an $(l, \kappa)-f M c$ set and $\eta(\underline{1}-v) \geq \imath, \eta^{*}(\underline{1}-v) \leq \kappa$.

Proof: (i) Let $\gamma$ is an $(l, \kappa)$-fMo set, and a crisp set $v \in I^{X}$ 
with $\eta(v) \geq \imath, \eta^{*}(v) \leq \kappa$, then

$$
\begin{aligned}
& \gamma \wedge v \leq\left(C_{\eta, \eta^{*}}\left(\theta I_{\eta, \eta^{*}}(\gamma, \imath, \kappa), \imath, \kappa\right)\right. \\
& \left.\vee I_{\eta, \eta^{*}}\left(\delta C_{\eta, \eta^{*}}(\gamma, \imath, \kappa), \imath, \kappa\right)\right) \wedge v \\
& =\left(C_{\eta, \eta^{*}}\left(\theta I_{\eta, \eta^{*}}(\gamma, \imath, \kappa), \imath, \kappa\right) \wedge v\right) \\
& \vee\left(I_{\eta, \eta^{*}}\left(\delta C_{\eta, \eta^{*}}(\gamma, \imath, \kappa), \imath, \kappa\right) \wedge v\right) \\
& \leq \quad\left(C_{\eta, \eta^{*}}\left(\theta I_{\eta, \eta^{*}}(\gamma, \imath, \kappa) \wedge v, \imath, \kappa\right)\right) \vee \\
& \left(I_{\eta, \eta^{*}}\left(\delta C_{\eta, \eta^{*}}(\gamma, \boldsymbol{\imath}, \kappa) \wedge \nu, \imath, \kappa\right)\right) \\
& \leq \quad\left(C_{\eta, \eta^{*}}\left(\theta I_{\eta, \eta^{*}}(\gamma \wedge v, \imath, \kappa), \imath, \kappa\right)\right) \vee \\
& \left(I_{\eta, \eta^{*}}\left(\delta C_{\eta, \eta^{*}}(\gamma \wedge v, \imath, \kappa), \imath, \kappa\right)\right)
\end{aligned}
$$

Hence $\gamma \wedge v$ is an $(\boldsymbol{\imath}, \kappa)-f M o$ set.

(ii) Similar to the proof of (i).

$\Xi$

Theorem 2.15. If $\gamma \in I^{X}$ is both $(\imath, \kappa)-f M o$ and $(l, \kappa)-f c$ set in $\left(X, \eta, \eta^{*}\right)$ then $\gamma$ is an $(l, \kappa)-f \theta$ so.

Proof: Let $\gamma$ be an $(\imath, \kappa)-f M o$ then

$$
\begin{aligned}
\gamma \leq & C_{\eta, \eta^{*}}\left(\theta I_{\eta, \eta^{*}}(\gamma, \imath, \kappa), \imath, \kappa\right) \\
& \vee I_{\eta, \eta^{*}}\left(\delta C_{\eta, \eta^{*}}(\gamma, \imath, \kappa), \imath, \kappa\right) \\
= & C_{\eta, \eta^{*}}\left(\theta I_{\eta, \eta^{*}}(\gamma, \imath, \kappa), \imath, \kappa\right) \vee I_{\eta, \eta^{*}}(\gamma, \imath, \kappa) \\
\leq & C_{\eta, \eta^{*}}\left(\theta I_{\eta, \eta^{*}}(\gamma, \imath, \kappa), \imath, \kappa\right) .
\end{aligned}
$$

Hence $\gamma$ is an $(\boldsymbol{\imath}, \kappa)-f \theta$ so.

Theorem 2.16. If $\gamma \in I^{X}$ is both $(\imath, \kappa)-f M c$ and $(\imath, \kappa)$ - $f o$ set in $\left(X, \eta, \eta^{*}\right)$ then $\gamma$ is an $(\imath, \kappa)-f \theta s c$.

Proof: Follows from theorem 2.15.

$\Xi$

Theorem 2.17. In a dfts $\left(X, \eta, \eta^{*}\right), \forall \gamma \in I^{X}$,

1. If $\eta(\gamma) \geq \imath$ and $\eta^{*}(\gamma) \leq \kappa$ then $\gamma$ is an $(\imath, \kappa)$-fMo set.

2. $I_{\eta, \eta^{*}}(\gamma, \imath, \kappa)$ is an $(\iota, \kappa)-f M o$ set.

3. $C_{\eta, \eta^{*}}(\gamma, \imath, \kappa)$ is an $(\imath, \kappa)-f M c$ set.

\section{An $(l, \kappa)$-generalized fuzzy $M$ closed sets}

Definition 3.1. In a $\left(X, \eta, \eta^{*}\right)$ be a dfts, $\gamma, v \in I^{X}$, a fuzzy set $\gamma$ is called an $(\boldsymbol{l}, \kappa)$ - generalized fuzzy $M$ closed (resp. $(\boldsymbol{l}, \boldsymbol{\kappa})$ generalized fuzzy $M$ open) (briefly $(\boldsymbol{\imath}, \kappa)-g f M c$ (resp. $(\boldsymbol{\imath}, \kappa)$ $g f M o)$ ) set if $M C_{\eta, \eta^{*}}(\gamma, \imath, \kappa) \leq v$ whenever $\gamma \leq v, \eta(v) \geq \imath$ and $\eta^{*} \leq \kappa$ (resp. $\underline{1}-\gamma$ is an $(\boldsymbol{l}, \kappa)-g f M c$ set $)$.

Theorem 3.2. In a dfts $\left(X, \eta, \eta^{*}\right), \gamma \in I^{X}$ is $(\imath, \kappa)$ - $g$ fMo set iff $v \leq M I_{\eta, \eta^{*}}(\gamma, \imath, \kappa)$ whenever $v \leq \gamma, \eta(\underline{1}-v) \geq \imath$ and $\eta^{*}(\underline{1}-v) \leq \kappa$.

Definition 3.3. In a dfts $\left(X, \eta, \eta^{*}\right), \forall \gamma \in I^{X}$, an $(\boldsymbol{\imath}, \kappa)$ - generalized fuzzy $M$ closure operator denoted as $(\imath, \kappa)-G M C_{\eta, \eta^{*}}$ : $I^{X} \times I_{0} \times I_{1} \rightarrow I^{X}$ defined as

$G M C_{\eta, \eta^{*}}(\gamma, \imath, \kappa)=\bigwedge\left\{v \in I^{X} \mid \gamma \leq v\right.$ and $v$ is $\left.(\imath, \kappa)-g f M c\right\}$
Theorem 3.4. In a dfts $\left(X, \eta, \eta^{*}\right), \forall \gamma, v \in I^{X}$, then the operator $(\imath, \kappa)-G M C_{\eta, \eta^{*}}$ satisfies the following statements

1. $G M C_{\eta, \eta^{*}}(\underline{0}, \imath, \kappa)=\underline{0}$, and $G M C_{\eta, \eta^{*}}(\underline{1}, \imath, \kappa)=\underline{1}$.

2. $\gamma \leq G M C_{\eta, \eta^{*}}(\gamma, \imath, \kappa)$

3. $G M C_{\eta, \eta^{*}}(\gamma \vee v, \imath, \kappa)$ $\geq G M C_{\eta, \eta^{*}}(\gamma, \imath, \kappa) \vee G M C_{\eta, \eta^{*}}(\nu, \imath, \kappa)$.

4. $G M C_{\eta, \eta^{*}}\left(G M C_{\eta, \eta^{*}}(\gamma, \imath, \kappa), \imath, \kappa\right)=G M C_{\eta, \eta^{*}}(\gamma, \imath, \kappa)$.

5. If $\gamma$ is $(\imath, \kappa)-g f M c$ set then $G M C_{\eta, \eta^{*}}(\gamma, \imath, \kappa)=\gamma$.

6. $G M C_{\eta, \eta^{*}}(\gamma, \imath, \kappa) \leq M C_{\eta, \eta^{*}}(\gamma, \imath, \kappa) \leq C_{\eta, \eta^{*}}(\gamma, \imath, \kappa)$.

Theorem 3.5. In a dfts $\left(X, \eta, \eta^{*}\right), \forall \gamma \in I^{X}$, an $(\imath, \kappa)$ - generalized fuzzy $M$ interior operator denoted as $(\imath, \kappa)-G M I_{\eta}, \eta^{*}$ : $I^{X} \times I_{0} \times I_{1} \rightarrow I^{X}$ given by

$G M I_{\eta, \eta^{*}}(\gamma, \imath, \kappa)=\bigvee\left\{v \in I^{X} \mid \gamma \geq v\right.$ and $v$ is $\left.(\boldsymbol{\imath}, \kappa)-g f M o\right\}$, and

$$
G M I_{\eta, \eta^{*}}(\underline{1}-\gamma, \imath, \kappa)=\underline{1}-G M C_{\eta, \eta^{*}}(\gamma, \imath, \kappa) .
$$

Proposition 3.6. In a dfts $\left(X, \eta, \eta^{*}\right)$, let an $(\imath, \kappa)-g f M c$ set $\gamma \in I^{X}$ and

1. if $\gamma$ is an $(\boldsymbol{\imath}, \kappa)-f M o$ set then $\gamma$ is an $(\boldsymbol{\imath}, \kappa)-f M c$ set.

2. if $\gamma$ is an $(\imath, \kappa)-f o$ set then $\gamma \wedge v$ is an $(\imath, \kappa)-f M c$ set whenever $v \leq M C_{\eta, \eta^{*}}(\gamma, \imath, \kappa)$.

Proof: (1) Let $\gamma$ be an $(\boldsymbol{\imath}, \kappa)-g f M c$ set and an $(\boldsymbol{\imath}, \kappa)-f M o$ set such that $\gamma \leq \gamma$.

$\Rightarrow M C_{\eta, \eta^{*}}(\gamma, \imath, \kappa) \leq \gamma$. Since $\gamma \leq M C_{\eta, \eta^{*}}(\gamma, \imath, \kappa)$,

$\Rightarrow \gamma=M C_{\eta, \eta^{*}}(\gamma, \boldsymbol{\imath}, \kappa)$. Therefore $\gamma$ is an $(\boldsymbol{\imath}, \kappa)-f M c$ set.

(2) Let $\gamma$ be an $(\boldsymbol{l}, \kappa)-g f M c$ set and an $(\boldsymbol{\imath}, \kappa)$ - $f o$ set, then

$$
\begin{aligned}
M C_{\eta, \eta^{*}}(\gamma, \imath, \kappa) \leq \gamma & \Rightarrow \gamma \text { is an }(\imath, \kappa)-f M c \text { set } \\
& \Rightarrow \gamma \wedge v \text { is an }(\imath, \kappa)-f M c \text { set } \\
& \Rightarrow \gamma \wedge v \text { is an }(\imath, \kappa)-g f M c \text { set. }
\end{aligned}
$$

Definition 3.7. In a dfts $\left(X, \eta, \eta^{*}\right)$, a fuzzy set $\gamma$ is called as $(\iota, \kappa)$-generalized* fuzzy $M$ closed (resp. $(\boldsymbol{\imath}, \kappa)$-generalized* fuzzy $M$ open) (briefly $(\imath, \kappa)-g^{*} f M c\left(\operatorname{resp} .(\imath, \kappa)-g^{*} f M o\right)$ ) set if $M C_{\eta, \eta^{*}}(\gamma, \boldsymbol{\imath}, \kappa) \leq v$ whenever $\gamma \leq \nu$ and $v$ is an $(\boldsymbol{\imath}, \kappa)$ $g$ fo set in $I^{X}$ (resp. $\underline{1}-\gamma$ is $(\boldsymbol{\imath}, \kappa)-g f M^{*} c$ set).

Theorem 3.8. In a dfts $\left(X, \eta, \eta^{*}\right)$, a fuzzy set $\gamma$ is $(l, \kappa)$ $g^{*} f M o$ set if and only if $v \leq M I_{\eta, \eta^{*}}(\gamma, \imath, \kappa)$ whenever $v$ is an $(\boldsymbol{\imath}, \kappa)-g f c$ set.

Proof: Let $\gamma$ be an $(\boldsymbol{\imath}, \kappa)-g^{*} f M o$ set in $I^{X}$, and let $v$ is an $(\boldsymbol{\imath}, \kappa)-g f c$ set $\ni v \leq \gamma$, by definition (3.1), $1-\gamma$ is an $(\boldsymbol{\imath}, \kappa)$ $g f o$ set in $I^{X} \& \underline{1}-\gamma \leq \underline{1}-v$. But $\underline{1}-\gamma$ is an $(\imath, \kappa)-g^{*} f M c$ set, then $M C_{\eta, \eta^{*}}(\underline{1}-\gamma, \imath, \kappa) \leq \underline{1}-v$. But

$$
M C_{\eta, \eta^{*}}(\underline{1}-\gamma, \imath, \kappa)=\underline{1}-M I_{\eta, \eta^{*}}(\gamma, \imath, \kappa) \leq \underline{1}-v .
$$


Hence, $v \leq M I_{\eta, \eta^{*}}(\gamma, \imath, \kappa)$.

Conversely, let $v \leq M I_{\eta, \eta^{*}}(\gamma, \imath, \kappa)$ whenever $v \leq \gamma$ and $v$ is an $(l, \kappa)-g f c$ set, now, $\underline{1}-M I_{\eta, \eta^{*}}(\gamma, \imath, \kappa) \leq \underline{1}-v$, Thus $M C_{\eta, \eta^{*}}(\underline{1}-\gamma, l, \kappa) \leq \underline{1}-v$. Therefore $\underline{1}-\gamma$ is an $(l, \kappa)$ $g f M c$ set. Hence $\gamma$ is an $(l, \kappa)-g f M o$ set.

Proposition 3.9. In a dfts $\left(X, \eta, \eta^{*}\right), \gamma \wedge v$ is an $(\imath, \kappa)-g^{*} f M c$ set if $\gamma \& v$ are $(\imath, \kappa)-g^{*} f M c$ sets.

Proof: Assume $\gamma$ and $v$ are $(l, \kappa)-g^{*} f M c$ sets, $\ni \gamma \wedge v \leq$ $v, \forall(l, \kappa)$-gfo set $v, M C_{\eta, \eta^{*}}(\gamma, \imath, \kappa) \leq v$, and since $\gamma$ is an $(\imath, \kappa)-g^{*} f M c$ set $M C_{\eta, \eta^{*}}(\gamma, \imath, \kappa) \leq v \forall(l, \kappa)$-gfo set $v \in I^{X}$ and $\gamma \leq v$. Also $v$ is an $(l, \kappa)-g^{*} f M c$ set $M C_{\eta, \eta^{*}}(v, l, \kappa) \leq$ $v \forall(l, \kappa)$-gfo set $v \in I^{X}, \& v \leq v$. Therefore

$$
M C_{\eta, \eta^{*}}(\gamma, \imath, \kappa) \wedge M C_{\eta, \eta^{*}}(\nu, \imath, \kappa) \leq v
$$

whenever $\gamma \wedge v \leq v$. Hence $\gamma \wedge v$ is an $(l, \kappa)-g^{*} f M c$ sets in $I^{X}$.

Proposition 3.10. In a dfts $\left(X, \eta, \eta^{*}\right), \gamma \wedge v$ is an $(l, \kappa)$ $g^{*} f M c$ set if $\gamma$ is $(\imath, \kappa)-g^{*} f M c$ set and $\eta(v) \geq \imath, \eta^{*}(v) \leq \kappa$.

Proof: Since every $(l, \kappa)-f c$ set is an $(l, \kappa)-g^{*} f M c$ set and from the proposition 3.9 we have the proof.

Proposition 3.11. In a dfts $\left(X, \eta, \eta^{*}\right), \gamma$ is an $(l, \kappa)-g^{*} f M c$ set and

1. if $\gamma$ is an $(l, \kappa)-g f o$ set then $\gamma$ is an $(l, \kappa)-f M c$ set.

2. if $\gamma \leq v \leq M C_{\eta, \eta^{*}}(\gamma, l, \kappa)$ then $v$ is an $(l, \kappa)-g^{*} f M c$ set.

Proof: (1) Suppose that $\gamma$ is both $(l, \kappa)-g f o$ and $(l, \kappa)-g^{*} f M c$ set in $I^{X}$. Since $\gamma$ is an $(l, \kappa)$-gfo set, $M C_{\eta, \eta^{*}}(\gamma, \boldsymbol{l}, \kappa) \leq \gamma$, since $\gamma$ is an $(l, \kappa)-g^{*} f M c$ set $\gamma \leq M C_{\eta, \eta^{*}}(\gamma, l, \kappa)$. Therefore $\gamma=M C_{\eta, \eta^{*}}(\gamma, \imath, \kappa)$.

(2) Suppose that $\gamma$ is an $(l, \kappa)-g^{*} f M c$ set and $v$ is an $(l, \kappa)$-gfo set in $I^{X} \ni \gamma \leq v \leq v$ and let $v \leq M C_{\eta, \eta^{*}}(\gamma, \imath, \kappa)$, This implies that

$$
\begin{aligned}
M C_{\eta, \eta^{*}}(v, \imath, \kappa) & \leq M C_{\eta, \eta^{*}}\left(M C_{\eta, \eta^{*}}(\gamma, \iota, \kappa), \iota, \kappa\right) \\
& =M C_{\eta, \eta^{*}}(\gamma, \imath, \kappa) .
\end{aligned}
$$

Since $\gamma$ is an an $(l, \kappa)-g^{*} f M c, v$ is an $(l, \kappa)-g f o$ and $\gamma \leq v$ we can say that $M C_{\eta, \eta^{*}}(\gamma, \imath, \kappa) v$, this implies that

$$
M C_{\eta, \eta^{*}}(v, \imath, \kappa) \leq v .
$$

Hence $v$ is an $(l, \kappa)-g^{*} f M c$ set.

Theorem 3.12. Let $\left(X, \eta_{1}, \eta_{1}^{*}\right)$ and $\left(Y, \eta_{2}, \eta_{2}^{*}\right)$ be dfts's. If $\gamma \leq \underline{1}_{Y} \leq \underline{1}_{X}$, $\ni \gamma$ is $(l, \kappa)-g^{*} f M c$ set in $I^{X}$, then $\gamma$ is an $(l, \kappa)-g^{*} f M c$ set relative to $Y$.

Proof: Suppose that $\left(X, \eta_{1}, \eta_{1}^{*}\right)$ and $\left(Y, \eta_{2}, \eta_{2}^{*}\right)$ be dfts's $\ni$ $\gamma \leq \underline{1}_{Y} \leq \underline{1}_{X}$, \& $\gamma \in I^{X}$ is $(l, \kappa)-g^{*} f M c$ set. And let $\gamma \leq$ $\underline{1}_{Y} \wedge v \ni \nu \in I^{X}$ is an $(l, \kappa)$-gfo set.

Since $\gamma \in I^{X}$ is $(l, \kappa)-g^{*} f M c$ set $\gamma \leq v \Rightarrow M C_{\eta, \eta^{*}}(\gamma, \imath, \kappa) \leq$ $v$. So that $\underline{1}_{Y} \wedge M C_{\eta, \eta^{*}}(\gamma, l, \kappa) \leq \underline{1}_{Y} \wedge v$. Therefore $\gamma$ is an $(l, \kappa)-g^{*} f M c$ set relative to $Y$.
Theorem 3.13. In a dfts $\left(X, \eta, \eta^{*}\right), \forall v \leq \gamma$, if $v$ is $(l, \kappa)$ $g^{*} f M c$ set relative to $\gamma \ni \gamma$ is both $(\boldsymbol{\imath}, \kappa)-g f o$ and $(\boldsymbol{\imath}, \boldsymbol{\kappa})$ $g^{*} f M c$ set of $I^{X}$ then $v$ is an $(l, \kappa)-g^{*} f M c$ set relative to $X$.

Proof: Let $v$ is an $(r, s)-g^{*} f M c$ set and $\eta(v) \geq \imath$ and $\eta^{*}(v)$ $\leq \kappa \ni \& v \leq v$. But we have, $v \leq \gamma \leq \underline{1}$, and so $v \leq \gamma v \leq v$, hence $v \leq \gamma \wedge v$. Therefore, $v$ is an $(l, \kappa)-g^{*} f M c$ relative to $\gamma$, i.e. $\gamma \wedge M C_{\eta, \eta^{*}}(v, \imath, \kappa) \leq \gamma \wedge v \Rightarrow \gamma \wedge M C_{\eta, \eta^{*}}(v, \imath, \kappa) \leq v$.

Thus $\left(\gamma \wedge M C_{\eta, \eta^{*}}(v, l, \kappa)\right) \vee\left(\underline{1}-M C_{\eta, \eta^{*}}(v, l, \kappa)\right) \leq v \vee$ $\left(\underline{1}-M C_{\eta, \eta^{*}}(v, \imath, \kappa)\right)$.

This implies that

$$
\gamma \vee\left(\underline{1}-M C_{\eta, \eta^{*}}(v, l, \kappa)\right) \leq v \vee\left(\underline{1}-M C_{\eta, \eta^{*}}(v, l, \kappa)\right) .
$$

Because $\gamma$ is an $(l, \kappa)-g^{*} f M c$ set, $M C_{\eta, \eta^{*}}(\gamma, \boldsymbol{l}, \kappa) \leq v \vee(\underline{1}-$ $v)$. And so $v \leq \gamma \Rightarrow M C_{\eta, \eta^{*}}(v, \imath, \kappa) \leq M C_{\eta, \eta^{*}}(\gamma, \imath, \kappa)$.

Therefore

$$
\begin{aligned}
M C_{\eta, \eta^{*}}(v, \imath, \kappa) & \leq M C_{\eta, \eta^{*}}(\gamma, \imath, \kappa) \\
& \leq v \vee\left(\underline{1}-M C_{\eta, \eta^{*}}(\nu, \imath, \kappa)\right) .
\end{aligned}
$$

Thus $M C_{\eta, \eta^{*}}(\gamma, \iota, \kappa) \leq v$, but $M C_{\eta, \eta^{*}}(\gamma, \iota, \kappa)$ is not contained in $\left(\underline{1}-M C_{\eta, \eta^{*}}(v, l, \kappa)\right)$. i.e. $v$ is an $(l, \kappa)-g^{*} f M c$ relative to $X$.

\section{Acknowledgment}

The authors thank the referees to give their valuable suggestions to make this as valuable.

\section{References}

[1] K. Atanassov, Intuitionistic Fuzzy sets, Fuzzy sets and system, 20(1)(1986),84-96.

[2] D. Coker, An introduction to Intuitionistic Fuzzy Topological spaces, Fuzzy Sets and Systems, 88(1997), 81-89.

[3] A. I. El-Maghrabi and M. A. Al-Johany, $M$ - Open set in Topological Spaces, Pioneer Journal of Mathematics and Mathematical Sciences, 4 (2) (2011), 213-308.

[4] A. I. El-Maghrabi and M. A. Al-Johany, New types of functions by $M$ - Open sets, Journal of Taibah University for Science, 7 (2013), 137-145.

[5] A. I. El-Maghrabi and M. A. Al-Johany, Some applications of $M$ - open set in Topological Spaces, Journal of King Saud University- Science, 26 (2014), 261-266.

[6] A. I. El-Maghrabi and M. A. Al-Johany, Further properties on $M$ - Continuity, Journal of Egyptain Mathematical Society, 22 (2014), 63-69.

[7] J. G. Garcia, S. E. Rodabaugh, Order-theoretic, topological, categorical redundancies of interval-valued sets, grey sets, vague sets, interval-valued "intuitionistic" sets, "intuitionistic" fuzzy sets and topologies, Fuzzy Sets and Systems, 156 (2005), 445-484.

${ }^{[8]}$ E.P. Lee, Y.B. Im, Mated fuzzy topological spaces, International Journal of Fuzzy Logic and Intelligent Systems, 11, (2001), 161-165. 
[9] F. M. Mohammed and A. Ghareeb, More on generalized $b$-closed sets in double fuzzy topological spaces, Songklanakarin J. Sci. Technol., 38 (1) (2016), 99-103.

[10] F. M. Mohammed, M. S. M. Noorani and A. Ghareeb, New notions from $(\alpha, \beta)$ - Generalized Fuzzy Pre-open sets, GU J. Sci., 30(1) (2017), 311-331.

[11] P. Periyasamy, V. Chandrasekar, G. Saravanakumar and A. Vadivel, Fuzzy $e$-closed and Generalized Fuzzy $e$-closed sets in Double Fuzzy Topological Spaces, Submitted.

[12] A. A. Ramadan, S. E. Abbas and Abd El-Latif, Compactness in intuitionistic fuzzy topological spaces, International Journal of Mathematics and Mathematical Sciences, 1, (2005), 19-32.

[13] S.K. Samanta, T.K. Mondal, On intuitionistic gradation of openness, Fuzzy Sets and Systems, 131, (2002), 323 336.

[14] B. Vijayalakshmi,S. Bamini, M. Saraswathi and A. Vadivel Fuzzy $M$-open sets in Sostak's Fuzzy topological spaces, Submitted.

[15] L. A. Zadeh, Fuzzy sets, Inform. Control, 8 (1965), 338353.

[16] A. M. Zahran, M. A. Abd-Allah and A. Ghareeb, Several Types of Double Fuzzy Irresolute Functions, International Journal of Computational Cognition, 8 (2) (2010), 19-23. 